\title{
Bioinformatic Analysis of DNA Methylation in Neural Progenitor Cell Models of Alcohol Abuse
}

\author{
Eileen N. Oni ${ }^{1} \cdot$ Ronald P. Hart ${ }^{1}$
}

Published online: 5 August 2016

(C) Springer International Publishing AG 2016

\begin{abstract}
Several recent publications sought to investigate the effects of ethanol treatment on models of central nervous system development, specifically through changes in DNA methylation. Regulation of DNA methylation causes a long-lasting, epigenetic change in the capacity of the genome to respond to developmental or metabolic stimuli. Changes in technologies for quantifying DNA methylation have increased the ability to identify and interpret potential effects of ethanol. Here, we review these recent studies in order to evaluate the detection technologies and bioinformatic analyses. Our evaluation finds that whole- or targetedgenome sequencing combined with bisulfite conversion of unmethylated $\mathrm{G}$ to $\mathrm{U}$ residues is now the standard for assessing genome-wide effects, and specific differentially methylated regions can be validated by one of the several widely available techniques. The acceptance of these technologies should help understand how ethanol leads to lifelong developmental or behavioral deficits and, perhaps, suggest therapies to reverse these effects.
\end{abstract}

Keywords Epigenetics $\cdot$ Fetal alcohol syndrome $\cdot$ Neural stem cells $\cdot$ Neural progenitor cells $\cdot$ Bioinformatics

This article is part of the Topical Collection on Bioinformatics and Stem Cell

Ronald P. Hart

rhart@rutgers.edu

1 Department of Cell Biology \& Neuroscience, Rutgers University, 604 Allison Road, Piscataway, NJ 08854, USA

\section{Introduction-Why Is DNA Methylation Important in Alcohol Abuse?}

During differentiation, DNA undergoes epigenetic remodeling to specify cell fate and regulate key developmental processes. This type of remodeling can become the first step on a path towards normal somatic differentiation or development of abnormality. Two important and well-studied examples of this are alcohol use disorders (AUD) and fetal alcohol syndrome (FAS). AUD is the third leading cause of death in the USA, contributing to about one in ten deaths a year. The estimated cost of treatment and the loss of productivity due to AUD are estimated to be $\$ 223.5$ billion annually $[2,6,26]$. Similarly, FAS is one of the leading causes of preventable birth defects and developmental disabilities and is estimated to cost an individual with FAS up to $\$ 2$ million over his or her lifetime, with the cost to the entire USA believed to be $\$ 4$ billion annually $[1,20]$. Many biological effects of AUD and FAS are found in the central nervous system (CNS). For example, ethanol has been found to have negative effects on hippocampal neurogenesis [8], regulating the expression profile of the key epigenetic regulator methyl $5^{\prime} \mathrm{C}-$ phosphate- $\mathrm{G} 3{ }^{\prime}$ ( $\mathrm{CpG}$ ) binding protein 2 (MeCP2), metabolism, and cycle genes important for stem cell differentiation and proliferation [10]. While much of the work involved in AUD inheritance and FAS in offspring has focused on the maternal contributions, there is also evidence for the role of paternal epigenetic influence, where children of fathers with AUD have higher risk of psychological disorders [23]. Studies have also associated chronic alcohol consumption with increase sperm DNA methylation within differentially methylated regions (DMRs) that are important in regulating growth factors [22]. For this review, however, we will focus on direct exposure of neuronal precursor cells to alcohol. 
Several recent publications sought to understand the epigenetic effects of the alcohol ethanol, specifically, the epigenetic regulation of neuronal stem cells, within the space of genomewide DNA methylation (known as the methylome). These articles provide a valuable lesson in how methylation epigenetic data derived from genome-wide studies can be prepared and analyzed. In this review, we will discuss the bioinformatic strategies in four key articles in their attempts to elucidate the epigenetic properties of ethanol regulation during early differentiation and neural stem cell (NSC) differentiation as models of FAS. Using the power of bioinformatics, one can identify large sets of genes and ultimately understand the mechanisms of alcohol exposure and DNA modification in AUD and FAS.

\section{Methods for DNA Methylation Analysis}

Techniques for assessing genome-wide DNA methylation have been evolving rapidly over the past few years. The goal is to survey sequences throughout the genome, particularly those in "CpG islands" and promoters, for the presence of 5methylcytosine $(5 \mathrm{mC})$ enrichment to identify DMRs that might be linked with epigenetic regulation of adjacent genes. The first genome-wide methods were modifications of protocols using microarrays with probes designed to sample the genome at a reasonably high density. For example, the commonly used NimbleGen arrays are designed to cover all known CpG-rich regions and transcription promoters by "tiling" 50 nucleotide (nt) probes with an average spacing of 50 base pairs (bp) of non-probed sequence separating each probed sequence. In some examples, sheared fragments of genomic DNA from the selected sample are immunoprecipitated with antibodies specific for $5 \mathrm{mC}$ and this methyl-enriched DNA is compared with unenriched DNA using contrasting probe dye colors. This is a cost-effective way to sample nearly half of all $\mathrm{CpG}$ islands and promoter regions on a microarray with the then-available probe densities. A similar approach uses Affymetrix promoterfocused microarrays. In this case, methylation is determined by comparing bisulfite-treated with untreated DNA. Bisulfite converts unmethylated $\mathrm{C}$ residues to $\mathrm{U}$ residues, so a relatively robust hybridization signal in the bisulfite-treated sample indicates a preponderance of methylation. More recently, nextgeneration sequencing technologies supplanted microarray methods due to a reduction in costs and the higher resolution of detecting individually methylated $\mathrm{C}$ residues. Wholegenome methyl-seq can be performed by comparing bisulfite-converted DNA with either an unconverted DNA sample or reference genome sequence as control. However, the reduced sequence complexity of DNA where all or most $\mathrm{C}$ residues have been converted to $\mathrm{T}$ can reduce the effective alignment with genome. Both to increase the alignment of converted sequence and to increase the sequencing depth for regions of interest, targeted bisulfite sequencing is available.
For example, Agilent uses a large number of beadimmobilized hybridization probes to enrich $\mathrm{CpG}$ islands, promoters, and related sequence as part of its SureSelect ${ }^{\mathrm{TM}}$ Methyl-Seq Enrichment System. In summary, the microarray-based methods have the advantage of being less expensive to run and simpler to interpret. The use of antibodies to immunoprecipitate methylated $\mathrm{C}$ residues is likely to be less sensitive than direct bisulfite sequencing. Sequencing has the advantage of single-base resolution but makes data analysis much more challenging. Many of these methods have been used to study ethanol-induced regulation of DNA methylation.

\section{Analysis of DNA Methylation in Models of Fetal Alcohol Exposure}

\section{Whole-Embryo Exposure}

An early whole-genome study of ethanol exposure utilized whole-embryo cultures of mouse [16]. Time-mated embryos were explanted 8.25 days after conception (E8.25) into sterile bottles containing medium and grown in a rotating system for up to $48 \mathrm{~h}$. This allowed for precise ethanol exposure. Following $4 \mathrm{~h}$ of pre-culture, the medium was adjusted to $88 \mathrm{mM}(400 \mathrm{mg} / \mathrm{dL})$ ethanol and the bottle was sealed. This dose is within the observed range but well above the mean for neonatal blood alcohol content as summarized in a recent review [3]. This allowed for evaluation of the effect of blood alcohol concentrations on fetuses during the key process of neural tube closure. Samples from the cultured embryos were classified as neural tube open (NTO) or closed (NTC). DNA was extracted and immunoprecipitated with a $5 \mathrm{mC}$-specific antibody, amplified, and compared with unprocessed DNA samples using NimbleGen arrays focusing on $\mathrm{CpG}$ islands and promoter regions. The authors categorized all available RefSeq promoters based on GC content within a defined region of $1300 \mathrm{bp}$ upstream and $500 \mathrm{bp}$ downstream of the transcription start site (TSS) into three groups: high, low, and intermediate $\mathrm{CpG}$ density (labeled as HCP, LCP, and ICP in their results). Initial analysis averaged methylation levels from multiple probes over each promoter region. Over all ethanol-treated embryos, the ratios of hypermethylated regions in each of the three promoter categories (HCP, LCP, and ICP) were significantly increased compared with control [16]. The ICP group exhibited an inverse relationship between promoter DNA methylation and adjacent messenger RNA (mRNA) expression levels, as determined by Affymetrix expression microarrays.

A finer analysis of the probe-level data focused on sequences with two consecutive probes defined as differentially methylated ( \pm 1.3 -fold changed). These data were fit to a linear mixed model to account for sources of experimental variability as well as random error. As with the region-based analysis described above, similar patterns of promoter numbers were found to 
have increased or decreased methylation, with $\sim 10 \%$ occurring in both NTC and NTO groups and $\sim 4 \%$ occurring in only one of the groups. Upon splitting these into low or high $\mathrm{CpG}$ regions, there were greater effects in the NTO group than the NTC group. High CpG regions had more genes with decreased expression and low $\mathrm{CpG}$ regions had more genes increased. Pathway analysis of these categorized gene sets identified affected functions including embryonic development, including genes specific for tissues such as heart, retina, sensory nervous system, and stem cell migration, all consistent with known phenotypes of FAS.

This study provided a valuable contribution by carefully classifying a major developmental phenotype (neural tube closure or failure to close) and comparing with subcategorized promoter regions based on $\mathrm{CpG}$ content. It is clear that the effects of fetal alcohol exposure may have been hidden if all promoters were considered as a single group. Furthermore, comparing and contrasting methylation status, averaged over an entire promoter region, versus the status identified with a more sophisticated model allowed for local changes in methylation (using consecutive probe modeling), obtaining more general and more promoter-specific results. The study combined the mapping of differentially methylated $\mathrm{CpG}$ islands and promoter regions with RNA expression analysis to link hypothesized effects of methylation or demethylation on gene expression. Furthermore, by classifying probed regions by CpG density, the authors were able to more precisely parse out biological effects linked with patterns of regulation.

\section{NSCs and Cell Cycle Control}

The chronic consumption of alcohol, especially during key growth stages such as pregnancy or adolescence, can lead to neurological damage [21]. This is believed to occur through alterations in DNA methylation of genes encoding controllers of cell cycle checkpoints such as cyclins $(\mathrm{Ccn})$, cyclindependent kinases (Cdks), and their associated inhibitors. To emulate fetal alcohol exposure and understand this dynamic, symmetrically dividing NSCs derived from embryonic stem cells were used as a model [9]. An ethanol concentration of $400 \mathrm{mg} / \mathrm{dL}(88 \mathrm{mM})$ was used due to the finding of in vitro effects similar to those previously observed with rat fetuses in vivo treated with $300 \mathrm{mg} / \mathrm{dL}(33 \mathrm{mM})$ ethanol. Concentrations greater than $400 \mathrm{mg} / \mathrm{dL}$ affected NSC proliferation and cell death [4]. Lower concentrations (100-320 mg/ $\mathrm{dL} ; 22-69 \mathrm{mM})$ had no significant effects.

Hicks et al. [9] hypothesized that ethanol induces epigenetic alterations of cell cycle genes. Fibroblast growth factor 2 (FGF2), a pro-mitotic factor, or transforming growth factor b1 (TGFb1), an anti-mitogenic factor, was included to regulate cell division in NSC cultures. Ethanol affects these compounds inversely; ethanol inhibits the effects of FGF2 and enhances the anti-mitogenic effects of TGFb1. To assess the changes in cell cycle kinetics over exposure time, ethanol-treated NSCs were labeled with bromodeoxyuridine (BrdU) during the addition of FGF2 or TGFb1. With the addition of ethanol (48 $\mathrm{h}$ in closed chambers with $400 \mathrm{mg} / \mathrm{dL}$ ethanol), FGF2 or TGFb1 treatment resulted in longer G1 phase. S phase increased by $65 \%$ in FGF2 with ethanol treatment while G2/M phases were affected by ethanol in TGFb1-treated cultures. The authors conclude that ethanol alters cell cycle only in the presence of either proor anti-mitotic stimuli [9]. There was no significant change in cell cycle phases due to ethanol alone.

To identify molecular pathways responsible for the cell cycle effects of ethanol exposure in the presence of mitotic regulators, mouse Affymetrix gene expression arrays were run [9]. Expression analysis with TGFb1-treated cultures identified 569 differentially regulated transcripts; 446 of them were downregulated $(78 \%)$. Within FGF2 treated cultures, ethanol caused a significant downregulation of 122 out of 195 differentially regulated transcripts $(63 \%)$, with the single greatest change in gene clusters related to DNA replication and checkpoint associated transcripts, including the mRNA encoding cyclin, a key G1/S checkpoint protein. Ethanol treatment in the presence of TGFb1 led to downregulation of transcripts with aggregated functions including regulation of the G1/S transition. The fact that ethanol treatment caused downregulation of a large proportion of genes and that many are associated with cell cycle control supports the hypothesis that ethanol may be affecting repressive epigenetic events such as methylation.

To analyze genome-wide DNA methylation of NSC cultures following ethanol treatment, $\mathrm{CpG}$ methylation was assayed using an Affymetrix Mouse Promoter-specific microarray probed with bisulfite-treated DNA [9]. This microarray includes probes for regions within 8-10 kilobase pairs $(\mathrm{kb})$ of the start codon for all RefSeq genes, with roughly three fourths of all $\mathrm{CpG}$ islands covered by more than ten probes. Probe signals exceeding that of controls (FGF2 treated cultures), and therefore rich in methylated sequences, were associated with the neighboring gene and filtered for being within $30 \mathrm{~kb}$ of a $\mathrm{CpG}$ island. Parameters for comparisons between methylation and gene expression required at least a threefold increase in methylation signatures and at least a twofold decrease in transcript expression. Eighty-three genes in total were both downregulated via microarray analysis and hypermethylated. The major gene clusters derived from this list included DNA metabolic and replication genes with $20 \%$ of all genes in these categories being hypermethylated.

To understand the physiological relevance of these transcript alterations in vivo, the expression of cell cycle mRNAs was assessed in cultures of isolated fetal subventricular cells, which include endogenous NSC. Similar expression levels for six out of ten transcripts were found, including downregulation of the proliferation marker Ki67 and cell cycle regulator Cdc2a. This largely validates the algorithm for selecting DMR candidates. The authors concluded that the presence of growth factors with 
ethanol alters cell cycle kinetics of NSCs at G1/S checkpoints through hypermethylation [9]. They further postulated that hypermethylation of $\mathrm{G} 1 / \mathrm{S}$ regulators restricts feed forward mechanism to maintain the continuation of the cycle.

While in many ways this study used a logically similar approach as that used by [16], the incorporation of bisulfite treatment instead of immunoprecipitation for $5 \mathrm{mC}$ is likely to be more precise as a tool to identify DMRs. Unfortunately, the Affymetrix microarray designed to probe promoter sequences excluded about $25 \%$ of all $\mathrm{CpG}$ islands and likely most adjacent sequences, termed "shores." It appears that one difficulty in analyzing combinations of methylation and expression datasets is that subtle methylation differences in subsets of sequence regions may be masked by applying uniform filtering to the entire dataset.

\section{Ethanol Regulation of Methylation in DRG NSC}

Another study [28] aimed to model the epigenetic effects of FAS in NSC. Previous work demonstrated alterations in DNA methylation of genes involved in cell growth, cell cycle, and cancer within mouse embryo cultures [16]. Past work had also demonstrated altered neural tube development, which may be due to the fact that ethanol can interrupt the methylation process by inhibiting methionine synthase, which would redirect the utilization of folate, and decrease DNA methyl transferase mRNA levels. [16] Zhou and colleagues established the epigenetic effects of ethanol during the neurogenesis of dorsal root ganglion (DRG) NSC, whose epigenetic changes have been previously characterized during differentiation [24, 25], hypothesizing that ethanol alters the DNA methylation profile of NSCs during development.

Rat DRG NSCs were plated in the absence of bFGF to promote differentiation, and then split into three groups: control, ethanol at $400 \mathrm{mg} / \mathrm{dL}(88 \mathrm{mM})$ for $6 \mathrm{~h}$, and 5 - azacytidine (5-azaC,a methylation inhibitor). The morphological characteristics of the ethanol-treated NSCs displayed reduced cellular migration in addition to changes in microtubule-associated protein (Map2) to Oct4 ratios. Cellular distribution of DNA methylation markers, specifically $5 \mathrm{mC}$ and DNA methyltransferase 1 (DNMT1), was altered by ethanol, suggesting that global nuclear methylation patterns were affected.

To identify DMRs, DNA samples from untreated differentiated cells or undifferentiated cells, with or without ethanol treatment, were immunoprecipitated with $5 \mathrm{mC}$ antibody and labeled with Cy5. Input DNA was prepared and labeled with Cy3. Pairs of immunoprecipitated and input samples were mixed and hybridized to NimbleGen RN34 plus CpG island microarrays, designed to detect 19,530 rat promoters and $\mathrm{CpG}$ islands per slide. Each probed region is covered by $\sim 50 \mathrm{nt}$ oligos with $\sim 50 \mathrm{bp}$ gaps between them. The promoters and islands overlap $1.3 \mathrm{~kb}$ upstream and $0.5 \mathrm{~kb}$ downstream from transcription start sites for 21,632 RefSeq genes. Ratios of Cy5 to Cy3 indicate the relative enrichment of $5 \mathrm{mC}$-immunoprecipitated DNA. Two approaches were taken to identify DMRs.

First, average-level analysis considered the mean of all probes near each transcription start site and defined hypermethylation as being 1.3-fold higher than the genome-wide mean methylation ratio. Promoters with 1.3-fold lower DNA methylation levels were considered hypomethylated. This approach was intended to avoid distal nucleotide noise and focused on binding site for transcription factors, in order to be compared to other human studies. Results from this analysis noted a decrease in the diversity of methylated genes that occurred during differentiation. One thousand one hundred forty-three genes changed in methylation status over differentiation. Ethanol prevented this change in 272 of those. More precisely, 344 genes change from moderate to either a hyper- or hypomethylation state. Ethanol prevented 97 genes from making this change, with 78 genes prevented from hypermethylation and 19 changed from hypomethylation to moderate status. Among the 78 genes where ethanol blocked hypermethylation, functional analysis showed that neuronal receptors were prominent. These patterns of DMRs indicate a widespread effect of ethanol on methylation during DRG differentiation.

As a second approach, probe-level analysis was used to observe higher-resolution methylation patterns in distal regions without diluting signals over the core promoter. This analysis extended to the entire set of gene promoters and $\mathrm{CpG}$ islands covered by the array. DNA regions with increased or decreased methylation levels were based on signal intensities using two consecutive probe analysis methods, where adjacent probes (on the same DNA strand with each probe covering $50 \mathrm{bp}$ and typically $50 \mathrm{bp}$ apart) were used to assess methylation. Adjacent genes were considered to be hyper- or hypomethylated if they had no fewer than two consecutive and consistently differentially methylated probes. A linear mixed model data analysis was used to account for response variability (population average, subject specific effects) as well as random effects. Following adjacent-probe analysis, 1054 (FDR $\leq 30 \%$ ) regions showed a significant difference in DNA methylation, with 681 having decreased methylation and 373 with increased methylation.

A complementary method was used to validate selected differentially methylated genes. A set of genes, chosen with both average-level differences and two consecutive probe-level differences, was validated using Sequenom MassARRAY. PCR primers were designed to assay 26 gene promoter regions with 48 amplicons covering nine CpGs per amplicon. Each primer pair included a $\mathrm{T} 7$ promoter sequence. These primers were used to amplify bisulfite-treated genomic DNA. Following in vitro transcription and nucleotide-specific RNA cleavage (MassCLEAVE), MALDI-TOF mass spectrometry was used to acquire spectral peaks to create methylation ratios based on the number of $\mathrm{C}$ residues representing methylated $\mathrm{CpGs}$ or $\mathrm{U}$ residues due to bisulfite conversion of unmethylated CpGs. 
Genes were defined as having a methylation difference if they had greater than $10 \%$ of at least one $\mathrm{CpG}$ unit altered and changes in the same direction for all $\mathrm{CpG}$ units within an amplicon. The Sequenom protocol validated 12 genes of the 26 predicted to be differentially methylated by $5 \mathrm{mC}$ immunoprecipitation and microarray. Overall, verified hypermethylation was found in genes such as insulin growth factor (Igf2), SRY-box containing gene 7 (Sox7), and cut-like 2 (Cux2) that are mediators of cell cycle progression, neuronal regulation, and stem cell patterning. From this study, the authors conclude that ethanol alters the DNA methylation program of stem cell and differentiation qualities of NSCs.

This study, while relying on the less-precise $5 \mathrm{mC}$ immunoprecipitation method for detecting DMRs, took advantage of a medium-throughput validation technique. Among the examples covered here, this was the first study to attempt the validation of a substantial set of DMRs. Unfortunately, the mass spectrometry-based method, while highly sensitive to measuring methylation proportions in each $\mathrm{CpG}$, is too expensive for most users and too difficult to scale up for a more complete validation series. Furthermore, the proportion of genes validated (12 out of 26) seems relatively small. This could indicate that the immunoprecipitation/microarray screening produced many false positives or that the validation method was not sufficiently sensitive to detect small changes in methylation. Since methyl immunoprecipitation methods are by their nature less precise than bisulfite methods, we assume that screening was the problem in this analysis. Alternatively, the 1.3 -fold cutoff may have been too close to background noise levels to reliably select candidates. No matter which explanation is true, the discordance between screening and validation shows that a recursive consideration of screening methods could have improved the choice of filtering methods.

\section{Use of hESC as Model of Early Development}

A study by Khalid et al. [14] used the differentiation of human embryonic stem cells (hESCs) to understand the epigenetic effects of FAS during early embryonic development. Since methylome-wide studies had not been previously reported in ethanol-treated hESCs and since ethanol had been reported to alter the cellular functions of regulatory genes involved in stem cell maintenance and differentiation [7, 12], this study served to identify and interpret methylation changes induced by ethanol. Embryonic stem cell lines $\mathrm{H} 1$ and $\mathrm{H} 9$ were treated with 20 or $50 \mathrm{mM}$ ethanol (90 or $225 \mathrm{mg} / \mathrm{dL}$ ), selected to range from levels equivalent to legal driving limits to those observed in alcoholics [13]. Cells were treated for 24 or $48 \mathrm{~h}$ in order to mimic prolonged exposures and the low ethanol elimination rates early in fetal development [3].

The goal of the study was to determine how ethanol affects both DNA methylation and gene expression in a coordinated mechanism. Gene expression studies began with total RNA from triplicate biological samples. Each sample was biotinylated. cRNA was labeled, purified and fragmented, and hybridized with the Affymetrix Human Genome U133 plus 2.0 Array. Genomic DNA was isolated and the percentage of $5 \mathrm{mC}$ marking was quantified using an ELISA-like technique. For methylation mapping, total genomic DNA was isolated and sent to a service lab (Zymo Research) for processing. DNA was treated with bisulfite to convert unmethylated $\mathrm{C}$ residues to $\mathrm{U}$ and then the DNA was fragmented for standard whole-genome sequencing. While details of bioinformatic sequence analysis were not provided, presumably an algorithm similar to Bismark [15] was used to align bisulfitetransformed sequences and count methylated nucleotides. A typical approach for this is to align with two separate $\mathrm{C} \rightarrow \mathrm{U}$ or $\mathrm{G} \rightarrow$ A substituted genome sequences, one to model methylation for each of the two strands. Results showed a global increase in methylation, particularly near transcription start sites (TSS) and CpG islands, in agreement with overall levels of methylation determined on unfractionated DNA. Ideally, increased methylation of promoters or nearby $\mathrm{CpG}$ islands should be associated with decreased mRNA expression levels.

Weighted gene co-expression network analysis was used to identify genes that are affected by ethanol using both differentiated (embryoid bodies) and undifferentiated stem cells [14]. Unsupervised hierarchical clustering noted two clusters (labeled as turquoise and light yellow in the study) that had similar gene expression in differentiated cells with ethanol as in undifferentiated with or without ethanol, providing evidence for repression. Following a Kyoto Encyclopedia of Genes and Genomes (KEGG) pathway analysis, the turquoise gene group associated with methyltransferase and the light yellow group with RNA polymerase, which may be repressed, suggesting mechanisms affected by ethanol exposure. To confirm that these patterns were due to methylation, a sample set of four of the top ten downregulated genes was assayed after treatment with ethanol or 5-azaC, which blocks methylation. All four genes exhibited a reversal of inhibition and increased expression over untreated control levels. While the sample size for validation was small, the high rate of agreement substantiates the analysis methods used for interpreting methylome-wide effects. Furthermore, this not only confirms that ethanol-induced methylation caused gene repression but also that basal levels of methylation may have been present.

Aggregating the genes with reduced mRNA expression and increased methylation of promoters or $\mathrm{CpG}$ islands, ethanol enriched the regulation of functional groups related to neuroactive ligand-receptor interaction, vascular smooth muscle contraction, calcium signaling pathways, and energy metabolism [14]. The authors argue that these may be related to stem cell pluripotency and differentiation. Selected example genes were tested by qPCR to confirm regulation but the changes, while significantly different in ethanol treatment, were relatively minor. 
Affected genes were screened as targets of regulation by specific transcription factors. Results indicated that regulated genes were associated with multiple transcription factor binding site motifs, most notably those related to stem cell maintenance or differentiation. These included FOX, SMAD, and POU family members. Overall the effects of ethanol on the differentiation and pluripotency factors in ESCs showed numerous alterations of the methylome of differentiated embryonic stem cells.

The use of bisulfite modification coupled with deep genome sequencing appears to be a step forward in assessing effects of ethanol on the methylome. While the article did not provide much detail on how sequencing data were analyzed, at least the DMRs identified by these methods were validated by reversal after 5-azaC treatment to block methylation. By combining methylation analysis with gene expression studies, the authors were able to apply pathway analysis and transcription factor binding site enrichment to determine patterns of response to ethanol.

\section{Ethanol Regulation of MeCP2}

An article by Liyanage et al. [18] attempted to shed light on the discrepancies between studies on the effects of ethanol on MeCP2 expression [5] while also understanding the molecular mechanisms of DNA methylation at regulatory elements in the MeCP2 gene. Ethanol-induced alterations of methylation can contribute to deregulating MeCP2 expression [17]. Previous work from this group found MeCP2 expression changes following DNA methylation at adjacent regulatory elements using murine embryonic-derived NSCs, which previously had been shown to express MeCP2 and were used as a model for neural development. In this study, the authors identified whether ethanol-induced expression changes of MeCP2 are due to DNA methylation of MeCP2.

Binge-, chronic-, and withdrawal-like ethanol conditions were used to study effects of ethanol exposure on MeCP2 regulation in forebrain-derived NSCs (NSCs). To study the methylation effects of alcoholic-like ethanol concentrations ( $70 \mathrm{mM} ; 320 \mathrm{mg} / \mathrm{dL}$ ), cells were treated during the onset of differentiation for $48 \mathrm{~h}$ (as a model of a binge effect) or 8 days (to mimic a chronic exposure). Additionally, withdrawal effects were studied 6 days after withdrawal of ethanol. In previous work, an altered cellular morphology was observed in ethanol-exposed, cultured glia and neurons; thus, the morphological state of NSC was measured in response to ethanol treatment. This was assessed through the expression of neural and glial markers as well as measurement of neurite outgrowth and glial size. There were no significant changes in the numbers of cell-type specific markers. However, secondary and tertiary neurite outgrowths were increased in the chronic and withdrawal conditions. Glial size was also affected in that a 1.5-fold increase was observed during chronic ethanol exposure, but there was no effect in the withdrawal condition. $\mathrm{MeCP} 2$ expression levels were upregulated during chronic and binge exposure (1.46-fold [ $p>0.05], 1.55$-fold $[p>0.001]$, respectively) while the withdrawal condition had a 1.38-fold reduction in MeCP2 transcripts.

To establish whether MeCP2 upstream regulatory elements (termed R1-R3) or intron 1 (R4-R6) are targeted by ethanol, targeted pyrosequencing was used to analyze DNA methylation at selected $\mathrm{CpG}$ sites. Briefly, genomic DNA was treated with bisulfite, selected regions were amplified, and the sequence was determined by pyrosequencing. Non-bisulfite-treated samples served as control. Methylation of MeCP2 promoter $\mathrm{CpG}$ regions was not specifically altered, but during the chronic exposure, there was slight hypermethylation in region 6 of intron 1 $(4.5 \%, p>0.05)$. Since previous work showed that even slight changes in DNA methylation caused significant changes in MeCP2 expression [19], this result was judged to be biologically meaningful. However, bisulfite pyrosequencing does not distinguish between $5 \mathrm{mC}$ and 5-hydroxymethyl cytosine $(5 \mathrm{hmC})$. Further analysis differentiated which type of methylation $(5 \mathrm{mC}$ vs. $5 \mathrm{hmC})$ was present on these regulatory elements using $5 \mathrm{hmC}$-specific antibody to immunoprecipitate enriched DNA fragments (hMeDIP) or $5 \mathrm{mC}$-specific MeDIP. $5 \mathrm{hmC}$ levels were enriched in $\mathrm{R} 1, \mathrm{R} 3$, and $\mathrm{R} 5$ in the chronic ethanol exposure. $5 \mathrm{mC}$ enrichment levels (associated with diminished expression) were reduced in R3 and R6. Similar effects were seen in the withdrawal condition, which suggests that reduced MeCP2 expression may be due to a combination of reduced $5 \mathrm{hmC}$ and increased $5 \mathrm{mC}$ in these regions.

The effects on global $5 \mathrm{mC}$ and $5 \mathrm{hmC}$ DNA methylation during ethanol exposure and withdrawal were quantified using a DNA dot blot analysis. The binge condition showed no change in global expression levels. Chronic ethanol exposure conditions supported previous pyrosequencing results, where the levels of $5 \mathrm{mC}$ were increased while the withdrawal conditions reduced $5 \mathrm{hmC}$ levels. These findings suggest that ethanol treatment affects global DNA methylation levels at both $5 \mathrm{mC}$ and $5 \mathrm{hmC}$ marks and that these marks are affected independently of one another.

Since MeCP2 is relatively highly expressed in neurons and since it has been associated with repression of genes adjacent to methylated $\mathrm{CpG}$ regions, it is conceivable that the regulation of MeCP2 may serve to underlie global effects of ethanol. More importantly, if ethanol causes slight changes in methylation of regulatory sequences controlling $\mathrm{MeCP} 2$ and this leads to a reduction in $\mathrm{MeCP} 2$ activity, there is a possibility of at least some severity of symptoms consistent with MeCP2 knockdown, such as developmental delay, autism, and related disorders [11]. Interestingly, other epigenetic regulators such as DNMT1 and DNMT3A have also been found to be regulated by alcohol exposure [27], suggesting the presence of a direct mechanistic link between alcohol and epigenetic regulation.

This study relied upon a targeted analysis of CpG-rich sequences known to regulate a specific gene. Using a combination of bisulfite treatment, PCR amplification of selected 
regions, and pyrosequencing, the authors were able to detect and quantify small changes in methylation rates for individual bases. It remains impractical to use these sorts of targeted approaches to examine the entire methylome, but as we saw previously for a mass-spec-based validation or a reversal with 5 -azaC, some sort of validation is essential for complementing genome-wide screenings.

\section{Recommendations and Future Directions}

Upon review of these recent technologies for assessing effects of ethanol on the methylome, it is clear that specific advances have been made. Whole-genome sequencing or at least CpGregion-targeted sequencing of bisulfite-treated DNA should now be considered to be the norm. Bioinformatic workflows have been established for analyzing such genome-wide datasets, such as the Bismark package [15] that was used as an example. Validation methods provide not only precise measurements of selected regulatory regions, but they also verify that choices made during data filtering were appropriate. It seems clear that with the current state of technology, wholegenome bisulfite sequencing or at least sequencing of selected genomic regions should be used for future studies to obtain the highest-resolution, most reliable data. Biological models of alcohol exposure in fetal brain precursors should consider the validity of the model (cells vs. tissue; monolayer cultures vs. organoids, for example) and the dosage and exposure schedule for alcohol treatment. Understanding the role of ethanol in the etiology of disorders such as AUD and FAS is important, and therefore, the techniques used to investigate specific mechanisms must be acceptable by the scientific community.

\section{Acknowledgments Supported by NIH 5U10AA008401.}

\section{Compliance with Ethical Standards}

Conflict of Interest On behalf of all authors, the corresponding author states that there is no conflict of interest.

Human and Animal Rights and Informed Consent This article does not contain any studies with human or animal subjects performed by any of the authors.

\section{References}

1. American Academy of Pediatrics. American Academy of Pediatrics. Committee on Substance Abuse and Committee on Children With Disabilities. Fetal alcohol syndrome and alcohol-related neurodevelopmental disorders. Pediatrics. 2000;106(2 Pt 1):358-61.

2. Bouchery EE, Harwood HJ, Sacks JJ, Simon CJ, Brewer RD. Economic costs of excessive alcohol consumption in the U.S., 2006. Am J Prev Med. 2011;41(5):516-24. doi:10.1016/j. amepre.2011.06.045.
3. Burd L, Blair J, Dropps K. Prenatal alcohol exposure, blood alcohol concentrations and alcohol elimination rates for the mother, fetus and newborn. J Perinatol. 2012;32(9):652-9. doi:10.1038/jp.2012.57.

4. Cheema ZF, West JR, Miranda RC. Ethanol induces Fas/Apo [apoptosis]-1 mRNA and cell suicide in the developing cerebral cortex. Alcohol Clin Exp Res. 2000;24(4):535-43.

5. Gangisetty O, Bekdash R, Maglakelidze G, Sarkar DK. Fetal alcohol exposure alters proopiomelanocortin gene expression and hypothalamic-pituitary-adrenal axis function via increasing MeCP2 expression in the hypothalamus. PLoS One. 2014;9(11): e113228. doi:10.1371/journal.pone.0113228.

6. Hannigan JH, Berman RF. Amelioration of fetal alcohol-related neurodevelopmental disorders in rats: exploring pharmacological and environmental treatments. Neurotoxicol Teratol. 2000;22(1): 103-11.

7. Hansson AC, Nixon K, Rimondini R, Damadzic R, Sommer WH, Eskay R, et al. Long-term suppression of forebrain neurogenesis and loss of neuronal progenitor cells following prolonged alcohol dependence in rats. Int J Neuropsychopharmacol. 2010;13(5):58393. doi:10.1017/S1461145710000246.

8. He J, Nixon K, Shetty AK, Crews FT. Chronic alcohol exposure reduces hippocampal neurogenesis and dendritic growth of newborn neurons. Eur J Neurosci. 2005;21(10):2711-20. doi:10.1111 j.1460-9568.2005.04120.x.

9. Hicks SD, Middleton FA, Miller MW. Ethanol-induced methylation of cell cycle genes in neural stem cells. J Neurochem. 2010;114(6): 1767-80. doi:10.1111/j.1471-4159.2010.06886.x.

10. Hicks SD, Miller MW. Effects of ethanol on transforming growth factor Betal-dependent and -independent mechanisms of neural stem cell apoptosis. Exp Neurol. 2011;229(2):372-80. doi:10.1016/j.expneurol.2011.03.003.

11. Hulbert SW, Jiang YH. Monogenic mouse models of autism spectrum disorders: common mechanisms and missing links. Neuroscience. 2016;321:3-23. doi:10.1016/j.neuroscience.2015.12.040.

12. Jang MH, Shin MC, Kim EH, Kim CJ. Acute alcohol intoxication decreases cell proliferation and nitric oxide synthase expression in dentate gyrus of rats. Toxicol Lett. 2002;133(2-3):255-62.

13. Jones AW, Sternebring B. Kinetics of ethanol and methanol in alcoholics during detoxification. Alcohol Alcohol. 1992;27(6):641-7.

14. Khalid O, Kim JJ, Kim HS, Hoang M, Tu TG, Elie O, et al. Gene expression signatures affected by alcohol-induced DNA methylomic deregulation in human embryonic stem cells. Stem Cell Res. 2014;12(3):791-806. doi:10.1016/j.scr.2014.03.009.

15. Krueger F, Andrews SR. Bismark: a flexible aligner and methylation caller for Bisulfite-Seq applications. Bioinformatics. 2011;27(11):1571-2. doi:10.1093/bioinformatics/btr167.

16. Liu Y, Balaraman Y, Wang G, Nephew KP, Zhou FC. Alcohol exposure alters DNA methylation profiles in mouse embryos at early neurulation. Epigenetics. 2009;4(7):500-11.

17. Liyanage VR, Rastegar M. Rett syndrome and MeCP2. Neruomol Med. 2014;16(2):231-64. doi:10.1007/s12017-014-8295-9.

18. Liyanage VR, Zachariah RM, Davie JR, Rastegar M. Ethanol deregulates $\mathrm{Mecp} 2 / \mathrm{MeCP} 2$ in differentiating neural stem cells via interplay between 5-methylcytosine and 5-hydroxymethylcytosine at the Mecp2 regulatory elements. Exp Neurol. 2015;265:102-17. doi:10.1016/j.expneurol.2015.01.006.

19. Liyanage VR, Zachariah RM, Rastegar M. Decitabine alters the expression of Mecp2 isoforms via dynamic DNA methylation at the Mecp2 regulatory elements in neural stem cells. Mol Autism. 2013;4(1):46. doi:10.1186/2040-2392-4-46.

20. Lupton C, Burd L, Harwood R. Cost of fetal alcohol spectrum disorders. Am J Med Genet C Semin Med Genet. 2004;127C(1): 42-50. doi:10.1002/ajmg.c.30015.

21. Miller MW. Brain development : normal processes and the effects of alcohol and nicotine / edited by Michael W. Miller. Oxford; New York: Oxford University Press; 2006. 
22. Ouko LA, Shantikumar K, Knezovich J, Haycock P, Schnugh DJ, Ramsay M. Effect of alcohol consumption on CpG methylation in the differentially methylated regions of H19 and IG-DMR in male gametes: implications for fetal alcohol spectrum disorders. Alcohol Clin Exp Res. 2009;33(9):1615-27. doi:10.1111/j.15300277.2009.00993.x.

23. Ozkaragoz T, Satz P, Noble EP. Neuropsychological functioning in sons of active alcoholic, recovering alcoholic, and social drinking fathers. Alcohol. 1997;14(1):31-7.

24. Singh RP, Cheng YH, Nelson P, Zhou FC. Retentive multipotency of adult dorsal root ganglia stem cells. Cell Transplant. 2009a;18(1):55-68.

25. Singh RP, Shiue K, Schomberg D, Zhou FC. Cellular epigenetic modifications of neural stem cell differentiation. Cell Transplant. 2009b;18(10):1197-211. doi:10.3727/096368909X124831 62197204

26. Stahre M, Roeber J, Kanny D, Brewer RD, Zhang X. Contribution of excessive alcohol consumption to deaths and years of potential life lost in the United States. Prev Chronic Dis. 2014;11:E109. doi:10.5888/pcd11.130293.

27. Tyler CR, Allan AM. Prenatal alcohol exposure alters expression of neurogenesis-related genes in an ex vivo cell culture model. Alcohol. 2014;48(5):483-92. doi:10.1016/j.alcohol.2014.06.001.

28. Zhou FC, Balaraman Y, Teng M, Liu Y, Singh RP, Nephew KP. Alcohol alters DNA methylation patterns and inhibits neural stem cell differentiation. Alcohol Clin Exp Res. 2011;35(4):735-46. doi:10.1111/j.1530-0277.2010.01391.x. 